\title{
Inhalt
}

\author{
Vorwort - V
}

1 Einleitung -1

2 Thematik und Übersicht -3

\section{Forschungstheoretischer Hintergrund}

3 Soziogenese und Ontogenese der Schriftsprache - 9

3.1 Entwicklungsdimensionen der Schriftsprache - 9

3.2 Soziale Dimension der Schriftsprachaneignung - 12

3.2.1 (Konzeptionelle) Schriftlichkeit versus (konzeptionelle) Mündlichkeit — 13

3.2.2 Geschriebener Standard und gesprochener Dialekt in der Deutschschweiz — 17

3.2.3 Normen als soziales Regulativ sprachlichen Handelns - 21

3.3 Individuelle Dimensionen der Schriftsprachaneignung - 36

3.3.1 Wissensvoraussetzungen - $\mathbf{3 7}$

3.3.2 Entwicklungsphasen der Schriftsprachaneignung - 39

3.3.3 Basisqualifikationen der (Schrift-)Sprachaneignung - 48

3.3.4 Rolle der Selbsteinschätzung - $\mathbf{5 1}$

3.3.5 Der umstrittene Kompetenzbegriff $-\mathbf{5 3}$

3.4 Zusammenfassung Kapitel $\mathbf{3}-\mathbf{5 5}$

$4 \quad$ Schreiben als Prozess -58

4.1 Von der Syntax zur Schreibhandlung - 58

4.2 Prozessaspekte und Modelle des Schreibens - 60

4.3 Zusammenfassung Kapitel $4-66$

$5 \quad$ Grundlagen und Formen der Sprachbetrachtung - 68

5.1 Zweidimensionalität und Doppelfunktionalität der Sprache - 68

5.2 Bedeutung der Metasprache für die Sprachaneignung - 71

5.3 Metasprache und Schriftsprachaneignung - 74

5.3.1 Metasprache versus Sprachbewusstsein - 74 
5.3.2 Einheiten metasprachlicher Reflexion - $\mathbf{7 8}$

5.3.3 Zwischen implizitem und explizitem Sprachwissen - $\mathbf{8 0}$

5.3.4 Sprachwissen und Orthographieerwerb - $\mathbf{8 4}$

$5.4 \quad$ Bestimmungsmerkmale und Referenzbereiche metasprachlicher Äusserungen $-\mathbf{8 8}$

5.5 Sprachhandeln und Sprachwissen als Kontinuum - 92

5.6 Zusammenfassung Kapitel $5-95$

6 Sprachliche Konstruktionen zwischen Produkt, Prozess und Sprachwissen — 99

6.1 Über welche Muster wird Sprache erworben? - 99

6.2 Zum Konstruktionsbegriff — 101

6.3 Literale Konstruktionen zwischen Prozess und Produkt des Schreibens - 105

6.3.1 Literale Prozeduren als transphrastische Konstruktionen - 106

6.3.2 Literale Konstruktionen als Regulativ im Schreibprozess - $\mathbf{1 1 0}$

6.4 Literale Konstruktionen zwischen Text und Sprachwissen — 111

6.5 Zusammenfassung Kapitel $6-113$

\section{Konzeptualisierung der Untersuchung}

$7 \quad$ Herleitung der Forschungsfragen 119

7.1 Terminologische Differenzierung für die empirische Untersuchung — 119

7.2 Konkretisierung der Fragestellung - 122

7.2.1 Zur Rolle der globalen Sprachkompetenz — 122

7.2.2 Untersuchung der Schreibhandlung anhand von drei Korpora - 123

7.2.3 Ausformulierung der Forschungsfragen und Untersuchungshypothesen - 125

7.2.4 Funktion der Integration der Altersdimension — 131

$8 \quad$ Operationalisierung der Variablen - 134

8.1 Variablen zum Schreibprozess (Teilkorpus A) - 134

8.1.1 Umfang der Revisionen: Produkt-Prozess-Ratio - 135

8.1.2 Schreibsalven - 136

8.2 Variablen zum explizierten Sprachwissen (Teilkorpus B) $\mathbf{1 4 0}$ 
8.2.1 Referenzbereiche als Grössen metasprachlicher Reflexion - $\mathbf{1 4 0}$

8.2.2 Herleitung der Codes und Subcodes für die Annotation — 141

8.3 Textvariablen (Teilkorpus C) -145

8.3.1 Positionierungsprozeduren - 145

8.3.2 Lexik, Grammatik und Orthographie — 148

8.3.3 Syntaktische Komplexität — 152

9 Erhebungsinstrumente und Methode -154

9.1 Dokumentation der Erhebungsinstrumente - 154

9.1.1 Erhebung allgemeiner Daten sowie des sozioökonomischen Status 154

9.1.2 Erhebung der globalen Sprachkompetenz (C-Test) - 157

9.1.3 Erhebung der Kompetenz in der Lexik, der Grammatik und der Orthographie 167

9.1.4 Erhebung des Textes - 171

9.1.5 Erhebung des Schreibprozesses durch Keystroke Logging - 173

9.1.6 Erhebung und Aufbereitung der Daten zum explizierten Sprachwissen - 175

9.1.7 Erhebung des Selbstkonzepts der Kinder — 178

9.1.8 Erhebung kognitiver Grundfähigkeiten - $\mathbf{1 8 0}$

9.2 Beschreibung der Datenerhebung — 181

9.2.1 Rekrutierung der teilnehmenden Kinder und Klassen — 181

9.2.2 Ablauf der einzelnen Erhebungen — 182

9.3 Methoden der Datenanalyse und untersuchte Gruppen — 183

9.3.1 Form und Auswertung der Daten — 184

9.3.2 Überblick über die untersuchten Probandengruppen 184

9.3.3 Übersicht über die statistischen Verfahren — 185

$10 \quad$ Korpus und Beschreibung der Stichprobe -188

$10.1 \quad$ Datenstruktur $\mathbf{1 8 8}$

10.2 Charakterisierung der Probanden — 189

10.2.1 Alter $-\mathbf{1 8 9}$

10.2.2 Sprachlicher Hintergrund $-\mathbf{1 8 9}$

10.2.3 Sozioökonomischer Status - 190

10.2.4 Selbstkonzept $\mathbf{1 9 2}$

10.2.5 Kognitive Grundfähigkeiten — 193

10.3 Kompetenzgruppen als Grundlage für die Datenanalyse - 195

10.3.1 Clusterbildung aufgrund der C-Test-Werte — 197

10.3.2 Bildung von Kompetenzgruppen -199 


\section{Untersuchungsbericht}

11 Auswertungen entlang der Hauptfragestellungen - 205

11.1 Ergebnisse zum Schreibprozess — 206

11.1.1 Zur Rolle des Selbstkonzepts im Tastaturschreiben — 206

11.1.2 Überarbeitungsintensität: Produkt-Prozess-Ratio - 208

11.1.3 Salvenumfang - 209

11.2 Ergebnisse zum explizierten Sprachwissen - 211

11.2.1 Deskriptive Kennwerte zu den Referenzbereichen - 213

11.2.2 Zur Abhängigkeit der Referenzbereiche von der

Altersgruppe - $\mathbf{2 1 4}$

11.2.3 Zur Abhängigkeit der Referenzbereiche von der globalen Sprachkompetenz — 214

11.2.4 Zum Verhältnis von Normbezug und Schreibhandlung - 218

11.2.5 Konstruktionen an den Rändern tradierter Normen — 227

11.3 Ergebnisse zu Positionierungsprozeduren in den Kindertexten -229

11.3.1 Positionierungsprozeduren der 4. und der

6. Klassenstufe - 231

11.3.2 Positionierungsprozeduren sprachstarker und sprachschwacher Kinder 234

11.4 Ergebnisse zu Lexik, Grammatik, Orthographie und Syntax 236

11.4.1 Lexikalische Varianz — 237

11.4.2 Grammatische Qualität — 241

11.4.3 Orthographische Qualität - 245

11.4.4 Ergebnisse zur syntaktischen Komplexität -250

12 Zusammenfassung und Diskussion -255

$13 \quad$ Fazit und Ausblick -268

\section{Anhang}

14 Bibliographie -273

15 Abbildungs- und Tabellenverzeichnis - 293

15.1 Abbildungsverzeichnis -293

15.2 Tabellenverzeichnis $-\mathbf{2 9 4}$ 
16 Erhebungsmaterial -296

16.1 Fragebogen für die Schüler und Schülerinnen - 296

16.2 Fragebogen für die Eltern - 305

17 Exemplarische Auszüge aus dem Datenkorpus - 311

$17.1 \quad$ Textkorpus - $\mathbf{3 1 1}$

17.2 Schreibprozess - $\mathbf{3 1 1}$

$17.3 \quad$ Gesprächskorpus - $\mathbf{3 1 2}$

Register 323 
